\title{
Transverse instability of gravity-capillary solitary waves
}

\author{
Boguk Kim ${ }^{1}$ and T. R. Akylas ${ }^{2}$ \\ ${ }^{1}$ Department of Mathematics \\ ${ }^{2}$ Department of Mechanical Engineering \\ Massachusetts Institute of Technology
}

\begin{abstract}
Gravity-capillary solitary waves of depression, that bifurcate at the minimum phase speed on water of finite or infinite depth, while stable to perturbations along the propagation direction, are found to be unstable to transverse perturbations on the basis of a long-wave stability analysis. This suggests a possible generation mechanism of the new class of gravitycapillary lumps recently shown to also bifurcate at the minimum phase speed.
\end{abstract}

\section{Introduction}

The stability of plane solitary waves to perturbations transverse to the direction of propagation was examined first by Kadomtsev \& Petviashvili [1] on the basis of a generalization of the classical Korteweg-de Vries (KdV) equation allowing for weak transverse variations. This model equation, now known as the Kadomtsev-Petviashvili (KP) equation, predicts that KdV gravity-capillary solitary waves on shallow water are unstable to transverse perturbations if surface tension is strong enough:

$$
T>\frac{1}{3} \rho g h^{2},
$$

where $T$ denotes the coefficient of surface tension, $h$ the water depth, $\rho$ the fluid density and $g$ the gravitational acceleration. When this condition is satisfied, moreover, the KP equation admits fully localized solitary-wave solutions, commonly referred to as 'lumps', which thus become the asymptotic states of the initial-value problem in two spatial dimensions [2].

In more recent work, Bridges [3] obtained a condition for transverse instability to long-wave perturbations of solitary-wave solutions of Hamiltonian partial differential equations formulated as multi-symplectic systems. When applied to 
the water-wave problem with gravity and possibly surface tension present, this condition implies instability if

$$
\frac{\partial I}{\partial V}<0,
$$

where $I$ is a certain quantity related to the total horizontal linear momentum, or impulse, of the solitary wave and $V$ denotes the wave speed. (Bridges [3] refers to $I$ as the impulse but this ignores the circulation of the solitary wave which is non-zero in general; see $\S 2$ below.)

In the case of pure gravity solitary waves, it was shown by Longuet-Higgins [4] that $V \partial I / \partial V=\partial \mathcal{E} / \partial V, \mathcal{E}$ being the total energy of the solitary wave, so an alternative form of the instability condition (1.2) is

$$
\frac{1}{V} \frac{\partial \mathcal{E}}{\partial V}<0 .
$$

Moreover, $\mathcal{E}$ is known to be an increasing function of $V$ in the weakly nonlinear limit, where the KdV equation applies, and also for finite-amplitude waves with steepness (amplitude-to-depth ratio) below $\epsilon=0.781$. Hence, according to (1.3), transverse instability of gravity solitary waves first arises when the wave steepness exceeds this critical value which happens to also mark the onset of superharmonic instability to longitudinal perturbations [5].

It is worth noting that (1.2) and (1.3) apply in the long-wave-disturbance limit and provide sufficient, but not necessary, conditions for instability. This is consistent with recent work by Kataoka \& Tsutahara [6], who re-visited, using perturbation expansions, the eigenvalue problem governing the transverse instability of gravity solitary waves of the KdV type to long-wave disturbances. While their leading-order solution recovers condition (1.3), at the next order they find that instability in fact sets in at a somewhat lower wave steepness, $\epsilon=$ 0.713 , than the critical value 0.781 furnished by (1.3); according to the refined stability criterion, therefore, transverse instability arises prior to longitudinal instability.

The present paper is concerned with the transverse instability of gravitycapillary solitary waves. Rather than the classical KdV solitary waves on shallow water, attention is focused on solitary waves of the wavepacket type that are possible on water of finite or infinite depth in the presence of both gravity and surface tension, and have been studied extensively in recent years (see Dias \& Kharif [7] for a review). Solitary waves of this kind bifurcate from linear sinusoidal wavetrains at the minimum gravity-capillary phase speed and, in the small-amplitude limit, resemble wavepackets whose wave envelope and crests travel at the same speed $[8,9]$. Out of the two symmetric solitary-wave solution branches that bifurcate at the minimum phase speed, the depression branch is stable to longitudinal perturbations [10], so it is natural to inquire into its stability with respect to transverse perturbations.

It is straightforward to show (see §2) that the form (1.3), in terms of the total energy $\mathcal{E}$, of the transverse-instability condition (1.2) obtained by Bridges [3] remains valid in the case of gravity-capillary solitary waves as well. This suggests that the solitary waves of interest here, which exist below the minimum 
gravity-capillary phase speed, are unstable to transverse perturbations, as $\mathcal{E}$ is expected to increase when the wave speed $V$ is decreased. In fact, for a portion of the depression solitary-wave solution branch in deep water for which computations of $\mathcal{E}$ are available [11], it is clear that $\partial \mathcal{E} / \partial V<0$, implying instability according to (1.3).

Here we examine in a systematic way the stability of gravity-capillary solitary waves of the wavepacket type to long-wave transverse perturbations and compute the instability growth rate, using the expansion procedure of Kataoka \& Tsutahara [6] with the added effect of surface tension. The entire branch of depression solitary waves on water of finite or infinite depth, while stable to longitudinal perturbations, turns out to be transversely unstable, and the instability growth rate increases with $\mathcal{E}$.

In analogy with transversely-unstable $\mathrm{KdV}$ solitary waves that give rise to KP lumps in the high-surface-tension regime (1.1), it is likely that the instability discussed here also results in the formation of gravity-capillary lumps, but of the type recently found by Kim \& Akylas [12] and Parau, Vanden-Broeck \& Cooker [13].

\section{Preliminaries}

It is customary in studies of gravity-capillary solitary waves of the wavepacket type (e.g., $[8,10,14,15])$ to introduce non-dimensional variables such that the wave speed $c$ is normalized to 1 and trace solitary-wave solution branches in terms of

$$
\alpha=\frac{g T}{\rho c^{4}}
$$

and an additional parameter, such as

$$
H=\frac{h \rho c^{2}}{T}
$$

that involves the water depth. For the purpose of discussing the transverse instability of these solitary waves, however, it proves more convenient to use as characteristic length and time scale, respectively, $(T / \rho g)^{1 / 2}$ and $\left(T / \rho g^{3}\right)^{1 / 4}$, which do not depend on $c$; solitary-wave solution branches are thus traced via the dimensionless wave speed

$$
V=c\left(\frac{\rho}{g T}\right)^{1 / 4}=\alpha^{-\frac{1}{4}},
$$

and the water-depth parameter

$$
D=h\left(\frac{\rho g}{T}\right)^{1 / 2}=H \alpha^{1 / 2} .
$$

Gravity-capillary solitary waves of the wavepacket type arise when the minimum phase speed occurs at a finite wavenumber $[8,9]$, and this is possible when condition (1.1) is not met, implying $D>\sqrt{3}$. 
As remarked earlier, the total energy $\mathcal{E}(V, D)$ of a solitary wave plays an important part in the stability analysis. In terms of the velocity potential $\bar{\phi}(\theta, z ; V, D)$ and the free-surface elevation $z=\bar{\eta}(\theta ; V, D)$, where $\theta=x-V t$, associated with a solitary wave travelling along $x$ with speed $V$ on water of depth $D(-D<z<\bar{\eta}), \mathcal{E}$ is given by

$$
\mathcal{E}=\mathcal{T}+\mathcal{V}_{G}+\mathcal{V}_{T}
$$

where

$$
\mathcal{T}=\frac{1}{2} \int_{-\infty}^{\infty} \mathrm{d} \theta \int_{-D}^{\bar{\eta}}\left(\bar{\phi}_{\theta}^{2}+\bar{\phi}_{z}^{2}\right) \mathrm{d} z
$$

denotes the kinetic energy,

$$
\mathcal{V}_{G}=\frac{1}{2} \int_{-\infty}^{\infty} \bar{\eta}^{2} \mathrm{~d} \theta
$$

the gravitational potential energy and

$$
\mathcal{V}_{T}=\int_{-\infty}^{\infty}\left\{\left(1+\bar{\eta}_{\theta}^{2}\right)^{1 / 2}-1\right\} \mathrm{d} \theta
$$

the potential energy due to surface tension.

In addition, the total horizontal momentum, or impulse, $\mathcal{I}(V, D)$ of the solitary wave is given by

$$
\mathcal{I}=\int_{-\infty}^{\infty} \mathrm{d} \theta \int_{-D}^{\bar{\eta}} \bar{\phi}_{\theta} \mathrm{d} z .
$$

From (2.9), upon integrating by parts in $\theta$, one then has

$$
\mathcal{I}=I+D \mathcal{C}
$$

where

$$
\mathcal{C}=\left.\bar{\phi}\right|_{\theta=-\infty} ^{\theta=\infty}
$$

is the circulation of the solitary wave [4] and

$$
I=-\left.\int_{-\infty}^{\infty} \mathrm{d} \theta \bar{\eta}_{\theta} \bar{\phi}\right|_{z=\bar{\eta}}
$$

is the quantity that enters the instability condition (1.2) derived by Bridges [3]. Note that, in water of finite depth, $\mathcal{C} \neq 0$ so $I$ is distinct from the true impulse in general.

Differentiating expression (2.12) for $I$ with respect to $V$ and combining the result with (2.10), it follows that

$$
\frac{\partial I}{\partial V}=\frac{\partial \mathcal{I}}{\partial V}-D \frac{\partial \mathcal{C}}{\partial V}=\left.\int_{-\infty}^{\infty} \mathrm{d} \theta\left(\bar{\eta}_{V} \bar{\phi}_{\theta}-\bar{\eta}_{\theta} \bar{\phi}_{V}\right)\right|_{z=\bar{\eta}} .
$$

For pure gravity solitary waves, it is known from Longuet-Higgins [4] that

$$
\frac{1}{V} \frac{\partial \mathcal{E}}{\partial V}=\frac{\partial \mathcal{I}}{\partial V}-D \frac{\partial \mathcal{C}}{\partial V},
$$


so condition (1.2) can be replaced by (1.3), as indicated earlier. This remains true, however, when surface tension is also present, since it can be readily verified by differentiating $(2.5)$ with respect to $V$ that

$$
\frac{1}{V} \frac{\partial \mathcal{E}}{\partial V}=\left.\int_{-\infty}^{\infty} \mathrm{d} \theta\left(\bar{\eta}_{V} \bar{\phi}_{\theta}-\bar{\eta}_{\theta} \bar{\phi}_{V}\right)\right|_{z=\bar{\eta}} .
$$

Hence, according to (2.13),

$$
\frac{\partial I}{\partial V}=\frac{1}{V} \frac{\partial \mathcal{E}}{\partial V}
$$

so conditon (1.2) is entirely equivalent to (1.3) in the case of gravity-capillary solitary waves as well.

\section{$3 \quad$ Long-wave stability analysis}

To diagnose the stability to long transverse perturbations of gravity-capillary solitary waves of the wavepacket type, one may appeal directly to condition (1.2), or equivalently condition (1.3) in view of (2.16). In addition, it is possible to deduce the associated instability growth rate in the long-wave limit from the theory of Bridges [3]. For the latter purpose, rather than specializing the general formalism to the problem at hand, we find it more instructive to directly tackle the stability eigenvalue problem via a long-wave expansion procedure analogous to the one followed by Kataoka \& Tsutahara [6] for pure gravity solitary waves. A similar approach was taken in Kim \& Akylas [16] for the transverse instability of solitary waves of the Benjamin equation.

Briefly, assuming that infinitesimal perturbations with wavenumber $\mu$ in the transverse $(y-)$ direction and growth rate $\lambda$ are present in the free-surface elevation $\eta$ and the potential $\phi$,

$$
\eta=\bar{\eta}+\hat{\eta}(\theta) \mathrm{e}^{\mathrm{i} \mu y+\lambda t}, \quad \phi=\bar{\phi}+\hat{\phi}(\theta, z) \mathrm{e}^{\mathrm{i} \mu y+\lambda t},
$$

we linearize the governing equations about the underlying solitary-wave state. The perturbation eigenfunctions $\hat{\phi}$ and $\hat{\eta}$ then satisfy the Helmholtz equation

$$
\hat{\phi}_{\theta \theta}+\hat{\phi}_{z z}=\mu^{2} \hat{\phi} \quad(-D<z<\bar{\eta})
$$

subject to the bottom condition

$$
\hat{\phi}_{z}=0 \quad(z=-D)
$$

and the following two conditions on the free surface of the solitary wave:

$$
\begin{gathered}
\mathcal{L}_{1}(\hat{\phi}, \hat{\eta})=-\lambda \hat{\eta} \quad(z=\bar{\eta}), \\
\mathcal{L}_{2}(\hat{\phi}, \hat{\eta})=-\lambda \hat{\phi}-\mu^{2} \frac{\hat{\eta}}{\left(1+\bar{\eta}_{\theta}^{2}\right)^{1 / 2}} \quad(z=\bar{\eta}),
\end{gathered}
$$


where

$$
\begin{gathered}
\mathcal{L}_{1}(\hat{\phi}, \hat{\eta}) \equiv\left(-\frac{\partial}{\partial z}+\bar{\eta}_{\theta} \frac{\partial}{\partial \theta}\right) \hat{\phi}+\left\{\frac{\mathrm{d} \bar{\phi}_{\theta}}{\mathrm{d} \theta}+\left(-V+\bar{\phi}_{\theta}\right) \frac{\partial}{\partial \theta}\right\} \hat{\eta} \\
\mathcal{L}_{2}(\hat{\phi}, \hat{\eta}) \equiv\left(-V+\bar{\phi}_{\theta}\right) \frac{\mathrm{d} \hat{\phi}}{\mathrm{d} \theta}+\left\{\left(-V+\bar{\phi}_{\theta}\right) \bar{\phi}_{\theta z}+\bar{\phi}_{z} \bar{\phi}_{z z}+1\right\} \hat{\eta}-\left\{\frac{\hat{\eta}_{\theta}}{\left(1+\bar{\eta}_{\theta}^{2}\right)^{3 / 2}}\right\}_{\theta} .
\end{gathered}
$$

The boundary conditions (3.4) and (3.5) result from linearizing the kinematic and dynamic free-surface conditions, respectively. Compared with Kataoka \& Tsutahara [6], the additional effects of surface tension in the dynamic condition are reflected in the last term in (3.7) and the $O\left(\mu^{2}\right)$ term in (3.5).

In the long-wave limit $(\mu \ll 1)$, the eigenvalue problem (3.2)-(3.5) is solved by expanding $\hat{\phi}, \hat{\eta}$ and the growth rate $\lambda$, which acts as the eigenvalue, in powers of $\mu$ :

$$
\begin{gathered}
\hat{\phi}=\hat{\phi}^{(0)}+\mu \hat{\phi}^{(1)}+\mu^{2} \hat{\phi}^{(2)}+\cdots, \quad \hat{\eta}=\hat{\eta}^{(0)}+\mu \hat{\eta}^{(1)}+\mu^{2} \hat{\eta}^{(2)}+\cdots, \\
\lambda=\mu \lambda_{1}+\mu^{2} \lambda_{2}+\cdots .
\end{gathered}
$$

As in Kataoka \& Tsutahara [6], the leading-order solution is readily shown to be

$$
\hat{\phi}^{(0)}=\bar{\phi}_{\theta}, \quad \hat{\eta}^{(0)}=\bar{\eta}_{\theta} .
$$

Proceeding to $O(\mu), \hat{\phi}^{(1)}$ and $\hat{\eta}^{(1)}$ then satisfy the forced problem

$$
\begin{gathered}
\hat{\phi}_{\theta \theta}^{(1)}+\hat{\phi}_{z z}^{(1)}=R \quad(-D<z<\bar{\eta}), \\
\hat{\phi}_{z}^{(1)}=0 \quad(z=-D), \\
\mathcal{L}_{1}\left(\hat{\phi}^{(1)}, \hat{\eta}^{(1)}\right)=r_{1} \quad(z=\bar{\eta}), \\
\mathcal{L}_{2}\left(\hat{\phi}^{(1)}, \hat{\eta}^{(1)}\right)=r_{2} \quad(z=\bar{\eta}),
\end{gathered}
$$

where

$$
R=0, \quad r_{1}=-\lambda_{1} \bar{\eta}_{\theta}, \quad r_{2}=-\lambda_{1} \bar{\phi}_{\theta} .
$$

Since (3.10) is a well-behaved solution of the corresponding homogeneous problem, the forcing terms (3.15) must satisfy a certain condition for the inhomogeneous boundary-value problem (3.11)-(3.14) to have a solution that behaves acceptably as $\theta \rightarrow \pm \infty$. This solvability condition can be derived by forming the inner product of the forced equation (3.11) with the well-behaved solution of the adjoint boundary-value problem, and then making use of Green's identity along with the boundary conditions (3.12)-(3.14) and the properties of the adjoint solution. As it parallels closely an analogous derivation in Kataoka \& Tsutahara [6], here we shall omit the details (see [17]) and simply state the solvability condition:

$$
\int_{-\infty}^{\infty} \mathrm{d} \theta \int_{-D}^{\bar{\eta}} \bar{\phi}_{\theta} R \mathrm{~d} z+\left.\int_{-\infty}^{\infty} \mathrm{d} \theta\left(r_{1} \bar{\phi}_{\theta}-r_{2} \bar{\eta}_{\theta}\right)\right|_{z=\bar{\eta}}=0 .
$$


For the forcing terms (3.15), this condition is trivially met and the wellbehaved solution of the problem (3.11)-(3.14) turns out to be

$$
\hat{\phi}^{(1)}=-\lambda_{1} \bar{\phi}_{V}, \quad \hat{\eta}^{(1)}=-\lambda_{1} \bar{\eta}_{V} .
$$

At the next order, $\hat{\phi}^{(2)}$ and $\hat{\eta}^{(2)}$ are governed again by a forced problem of the form (3.11)-(3.14) but with forcing terms

$$
R=\bar{\phi}_{\theta}, \quad r_{1}=-\lambda_{2} \bar{\eta}_{\theta}+\lambda_{1}^{2} \bar{\eta}_{V}, \quad r_{2}=-\lambda_{2} \bar{\phi}_{\theta}+\lambda_{1}^{2} \bar{\phi}_{V}-\frac{\bar{\eta}_{\theta}}{\left(1+\bar{\eta}_{\theta}^{2}\right)^{1 / 2}} .
$$

Inserting the forcing terms (3.18) in the solvability condition (3.16) now yields a non-trivial result,

$$
\left.\lambda_{1}^{2} \int_{-\infty}^{\infty} \mathrm{d} \theta\left(\bar{\eta}_{V} \bar{\phi}_{\theta}-\bar{\eta}_{\theta} \bar{\phi}_{V}\right)\right|_{z=\bar{\eta}}+\int_{-\infty}^{\infty} \mathrm{d} \theta \int_{-D}^{\bar{\eta}} \bar{\phi}_{\theta}^{2} \mathrm{~d} z+\int_{-\infty}^{\infty} \frac{\bar{\eta}_{\theta}^{2}}{\left(1+\bar{\eta}_{\theta}^{2}\right)^{1 / 2}} \mathrm{~d} \theta=0
$$

which, taking into account (2.15), furnishes the following expression for the growth rate of long transverse perturbations to leading order in $\mu$ :

$$
\lambda_{1}^{2}=-\left(\int_{-\infty}^{\infty} \mathrm{d} \theta \int_{-D}^{\bar{\eta}} \bar{\phi}_{\theta}^{2} \mathrm{~d} z+\int_{-\infty}^{\infty} \frac{\bar{\eta}_{\theta}^{2}}{\left(1+\bar{\eta}_{\theta}^{2}\right)^{1 / 2}} \mathrm{~d} \theta\right) / \frac{1}{V} \frac{\partial \mathcal{E}}{\partial V}
$$

This confirms that (1.3) is a sufficient condition for transverse instability of solitary waves in the presence of gravity and surface tension.

As remarked earlier, in the case of pure gravity solitary waves, the instability condition (1.3) is first met when the wave steepness exceeds a certain critical value [5]; in order to gain information regarding the transverse instability of less steep solitary waves, it is necessary to carry the long-wave expansions (3.8) and (3.9) to higher order, as was done in Kataoka \& Tsutahara [6]. Fortunately, in the problem of interest here, as verified below, the leading-order result (3.20) predicts instability $\left(\lambda_{1}^{2}>0\right)$ for the entire solution branch of depression gravitycapillary solitary waves, so no higher-order analysis is needed.

\section{Results}

We now proceed to verify that $\partial \mathcal{E} / \partial V<0$ for gravity-capillary solitary waves of depression and compute the associated instability growth rate. To this end, we shall make use of small-amplitude expansions in the vicinity of the bifurcation point, slightly below the minimum phase speed, and fully numerical computations in the finite-amplitude regime.

In terms of the non-dimensional variables used here, for a given value of the water-depth parameter $D>\sqrt{3}$, the minimum gravity-capillary phase speed $V=V_{0}$ and the corresponding wavenumber $k_{0}$ are determined from the equation system

$$
V_{0}^{2}=\frac{1+k_{0}^{2}}{k_{0}} \tanh k_{0} D
$$




$$
\frac{1-k_{0}^{2}}{k_{0}} \tanh k_{0} D-\frac{D\left(1+k_{0}^{2}\right)}{\cosh ^{2} k_{0} D}=0 .
$$

In the deep-water limit $(D \rightarrow \infty)$, in particular,

$$
V_{0} \rightarrow \sqrt{2}, \quad k_{0} \rightarrow 1 .
$$

For $V$ slightly less than $V_{0}$, the bifurcating solitary-wave solution branches can be computed using perturbation expansions in terms of $\delta \equiv V_{0}-V(0<$ $\delta \ll 1$ ), and details are given in $[8,11]$. Specifically, in the case of deep water, the leading-order results for the depression solitary-wave branch are

$$
\begin{gathered}
\bar{\eta}=-\frac{8}{2^{1 / 4} \sqrt{11}} \delta^{1 / 2} \operatorname{sech}\left(2^{3 / 4} \delta^{1 / 2} \theta\right) \cos \theta+\cdots, \\
\bar{\phi}=-\frac{2^{1 / 4} 8}{\sqrt{11}} \delta^{1 / 2} \mathrm{e}^{z} \operatorname{sech}\left(2^{3 / 4} \delta^{1 / 2} \theta\right) \sin \theta+\cdots .
\end{gathered}
$$

Inserting (4.4) and (4.5) in (2.6)-(2.8), we find that

$$
\begin{aligned}
\mathcal{T} & \sim \frac{32}{2^{1 / 4} 11} \delta^{1 / 2}, \\
\mathcal{V}_{G} & \sim \frac{16}{2^{1 / 4} 11} \delta^{1 / 2}, \\
\mathcal{V}_{T} & \sim \frac{16}{2^{1 / 4} 11} \delta^{1 / 2} ;
\end{aligned}
$$

hence,

$$
\mathcal{E} \sim \frac{64}{2^{1 / 4} 11} \delta^{1 / 2} .
$$

As expected, $\mathcal{E}$ increases as $V$ is decreased below $V_{0}(\delta>0)$, so $\partial \mathcal{E} / \partial V<0$, and small-amplitude solitary waves on deep water are unstable to transverse perturbations. Similarly, from (3.20), making use of (4.4) and (4.5), it follows that

$$
\lambda_{1} \sim 2^{3 / 4} \delta^{1 / 2} .
$$

Therefore, combining (4.10) and (3.9), the instability growth rate of longwave transverse perturbations to small-amplitude depression solitary waves, travelling with speed $V$ slightly below the minimum phase speed $V_{0}=\sqrt{2}$ in deep water, is given by

$$
\lambda \sim 2^{3 / 4}\left(V-V_{0}\right)^{1 / 2} \mu,
$$

$\mu \ll 1$ being the perturbation wavenumber.

Figure 1 compares the asymptotic estimates (4.9) and (4.10) against values of $\mathcal{E}$ and $\lambda_{1}$, obtained from (2.5)-(2.8) and (3.20) using numerically-computed solitary-wave profiles close to the bifurcation point $V_{0}=\sqrt{2}$ in deep water. Not unexpectedly, the agreement is good only in the immediate vicinity of the bifurcation point. Numerical computations, nevertheless, indicate that $\partial \mathcal{E} / \partial V$ 
continues to be negative in the finite-amplitude regime, confirming the presence of instability, as illustrated in figure 2 for $D=2.5$ and $D \rightarrow \infty$. Moreover, the instability growth rate increases rapidly with the solitary-wave steepness, and the instability is stronger $\left(\lambda_{1}\right.$ is larger $)$ in the deep-water limit $(D \rightarrow \infty)$, at least for long-wave perturbations $(\mu \ll 1)$.

Solitary-wave profiles and the corresponding values of $\mathcal{E}$ and $\lambda_{1}$ were computed via a boundary-integral method, similar to the one described in [14], combined with Chebyshev spectral collocation (see [17] for details). Table 1 illustrates the convergence of $\mathcal{E}$ as the number of grid points $N$ is varied, for different values of the wave speed $V$ in finite depth $(D=2.5)$ and in deep water $(D \rightarrow \infty)$. Our computations of $\mathcal{E}$ also are consistent with those presented in [11] for a portion of the depression solitary-wave branch in deep water. The results shown in figure 1 were computed using $N=2048$, while those shown in figure 2 using $N=512$.

\section{Concluding remarks}

The preceding analysis has demonstrated that depression gravity-capillary solitary waves on water of finite or infinite depth, while stable to longitudinal perturbations [10], are unstable to transverse perturbations, similarly to elevation solitary waves of the Benjamin equation [16]. In the latter case, it is known that transverse instability results in the formation of fully locally confined solitary waves ('lumps') of elevation, that also bifurcate at the minimum phase speed [16]. In analogy, we expect that the instability discussed here leads to depression lumps of the type recently found in $[13,17]$.

As it is based on a long-wave expansion, the stability analysis presented here cannot furnish the maximum instability growth rate which corresponds to a finite-wavenumber perturbation $(\mu=O(1))$, in general. This task would require solving the eigenvalue problem (3.2)-(3.5) by numerical means. We remark that, for waves in the gravity-capillary regime, viscous effects may not be negligible, and, in order to assess the physical significance of transverse instability, it would be useful to compare the maximum instability growth rate against the decay rate caused by viscous dissipation, as was done in [10] for longitudinal perturbations. Based on the results shown in figure 2 for long-wave transverse perturbations, however, it would appear that transverse instability is quite strong, particularly for steep solitary waves, and warrants further theoretical and experimental study.

\section{Acknowledgements}

We wish to thank Professor Victor Shrira for helpful discussions. This work was supported by the Air Force Office of Scientific Research, Air Force Materials Command, USAF, under Grant Number FA9950-04-1-0125 and by the National Science Foundation Grant Number DMS-0305940. 
(a)

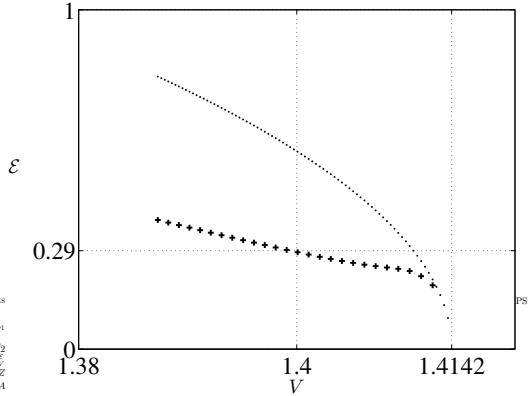

(b)

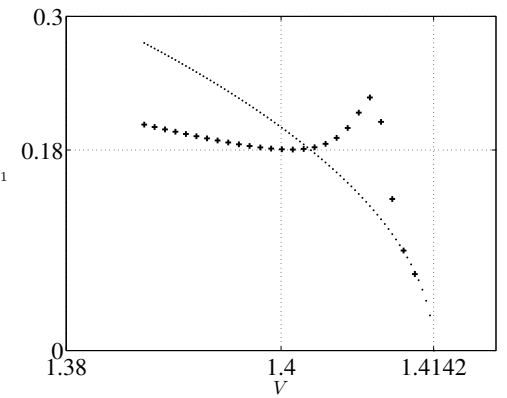

Figure 1: Comparison between computed results $(+)$ and the asymptotic estimates (4.9)-(4.10) ( $\cdots)$ for gravity-capillary solitary waves of speed $V$ near the bifurcation point $V_{0}=\sqrt{2}$ in deep water. (a) energy; (b) instability growth rate $\lambda_{1}$.

(a)
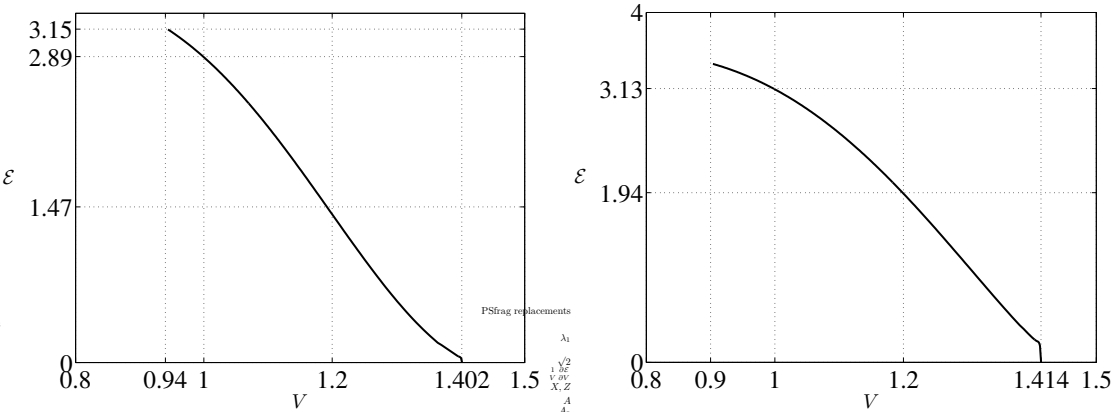

(b)
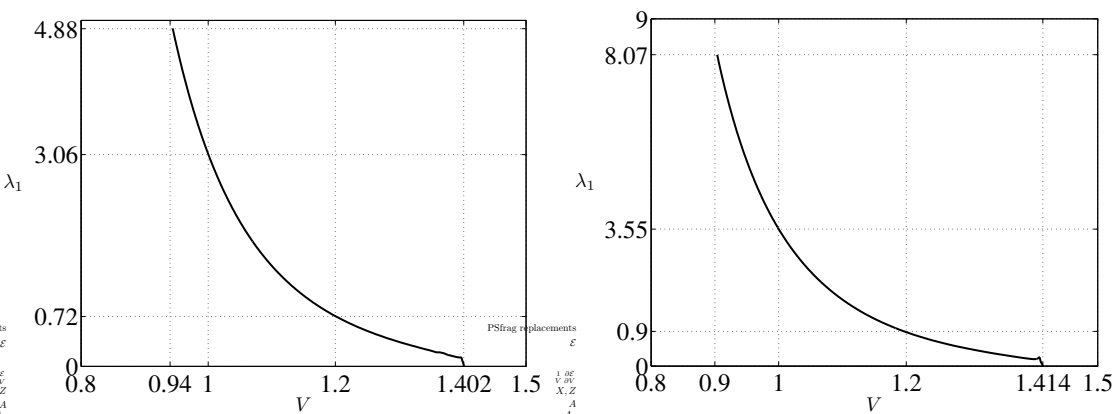

Figure 2: Energy $\mathcal{E}$ and instability growth rate $\lambda_{1}$ of depression gravity-capillary solitary waves as functions of wave speed $V$. Left column: finite depth $(D=2.5$, $\left.V_{0}=1.402\right)$. Right column: infinite depth $\left(D \rightarrow \infty, V_{0}=\sqrt{2}\right)$. (a) energy; (b) instability growth rate. 


\begin{tabular}{|c|c|c|c|c|c|c|}
\hline \multicolumn{3}{|c|}{$D=2.5$} & & \multicolumn{3}{|c|}{$D \rightarrow \infty$} \\
\hline$V=1.0$ & 1.2 & 1.4 & $N$ & $V=1.0$ & 1.2 & 1.4 \\
\hline 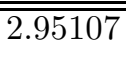 & 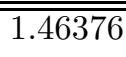 & (0.0230997 & $\overline{\overline{512}}$ & "3.12468 & $\bar{~} 1.93688$ & 0.284594 \\
\hline 2.95231 & 1.46579 & 0.0232001 & 1024 & 3.12650 & 1.94051 & 0.285844 \\
\hline 2.95269 & 1.46615 & 0.0232135 & 1536 & 3.12711 & 1.94171 & 0.286144 \\
\hline 2.95288 & 1.46642 & 0.0232221 & 2048 & 3.12741 & 1.94230 & 0.286265 \\
\hline
\end{tabular}

Table 1: Convergence of energy $\mathcal{E}$ as the number of grid points $N$ is varied for different values of the wave speed $V$ in finite depth $(D=2.5)$ and in deep water $(D \rightarrow \infty)$.

\section{References}

[1] Kadomtsev, B. B. \& Petviashvili, V. I. 1970 On the stability of solitary waves in a weakly dispersing medium. Sov. Phys. Dokl. 15, 539-541.

[2] Ablowitz, M. J \& Segur H. 1979 On the evolution of packets of water waves. J. Fluid Mech. 92, 691-715.

[3] BRidges, T.J. 2001 Transverse instability of solitary-wave states of the water-wave problem. J. Fluid Mech. 439, 255-278.

[4] Longuet-Higgins, M. S. 1974 On the mass, momentum, energy and circulation of a solitary wave. Proc. R. Soc. Lond. A 337, 1-37.

[5] Tanaka, M. 1986 The stability of solitary waves. Phys. Fluids 29, 650655.

[6] Kataoka, T. \& Tsutahara, M. 2004 Transverse instability of surface solitary waves. J. Fluid Mech. 512, 211-221.

[7] Dias, F. \& Kharif, C. 1999 Nonlinear gravity and capillary-gravity waves Annu. Rev. Fluid Mech. 31, 301-346.

[8] Akylas, T. R. 1993 Envelope solitons with stationary crests. Phys. Fluids A 5, 789-791.

[9] Longuet-Higgins, M. S. 1993 Capillary-gravity waves of solitary type and envelope solitons on deep water. J. Fluid Mech. 252, 703-711.

[10] Calvo, D. C. \& Akylas, T. R 2002 Stability of steep gravity-capillary solitary waves in deep water. J. Fluid Mech. 452, 123-243.

[11] Longuet-Higgins, M. S. 1989 Capillary-gravity waves of solitary type on deep water. J. Fluid Mech. 200, 451-470. 
[12] Kim, B. \& Akylas, T. R. 2005 On gravity-capillary lumps. J. Fluid Mech. 540, 337-351.

[13] Parau, E., Vanden-Broeck, J.-M \& Cooker, M. J. 2005 Nonlinear three-dimensional gravity-capillary solitary waves. J. Fluid Mech. 536, 99105.

[14] Dias, F., Menasce, D. \& Vanden-Broeck J.-M. 1996 Numerical study of capillary-gravity solitary waves. Euro. J. Mech. B 15, 17-36.

[15] Vanden-Broeck, J.-M. \& Dias, F. 1992 Gravity-capillary solitary waves in water of infinite depth and related free-surface flows. J. Fluid Mech. 240, 549-557.

[16] Kim, B. \& Akylas, T. R. 2006 On gravity-capillary lumps. Part 2. Twodimensional Benjamin equation. J. Fluid Mech. 557, 237-256.

[17] KIm, B. 2006 Doctoral dissertation, Three-dimensional solitary waves in dispersive wave systems, Department of Mathematics, MIT. 